\title{
Generation of a broadband continuum by a Ti:sapphire femtosecond oscillator with a 1-GHz repetition rate
}

\author{
A. Bartels and H. Kurz \\ Institut für Halbleitertechnik, RWTH Aachen, Sommerfeldstrasse 24, D-52074 Aachen, Germany
}

\begin{abstract}
A five-element Ti:sapphire femtosecond ring oscillator emitting a broadband continuum that ranges from 560 to $1150 \mathrm{~nm}$ at $-50 \mathrm{~dB}$ below the maximum with a repetition rate of $1 \mathrm{GHz}$ is demonstrated. The key element is a slightly convex cavity mirror that increases the self-amplitude modulation of a short pulse inside the resonator. Flat negative intracavity group-delay dispersion is required only for the core spectral part of the pulse. We believe that the device presented will make optical frequency metrology and future optical atomic clocks simpler and more stable. Within the reported ultrabroad spectrum a distinct strong emission band near $655 \mathrm{~nm}$ occurs that can be extracted to a powerful femtosecond pulse source far out of the amplification of Ti:sapphire.
\end{abstract}

Mode-locked femtosecond lasers with repetition rates in the gigahertz range ${ }^{1}$ have proved to be a key element in high-precision optical frequency metrology and have facilitated efforts to achieve improved atomic clocks based on optical transitions in the petahertz range. ${ }^{2-5}$ By virtue of its unique frequency comb properties, the laser serves as an optical clockwork for phase coherent upconversion of a reference microwave signal into the optical region or downconversion of a reference optical signal into an electronically countable microwave signal. Aside from having access to the easily measurable repetition rate, the crucial task is to measure the second degree of freedom of the frequency comb, i.e., its carrier envelope's offset frequency $f_{\mathrm{CEO}}$. The general technique for gaining access to $f_{\text {CEO }}$ was demonstrated by Reichert et al., who employed a 44-THz-wide frequency comb in conjunction with several auxiliary continuous-wave lasers. ${ }^{6}$ Because of the availability of much broader spectra, however, it has recently been possible to measure and control $f_{\text {CEO }}$ by use of self-referencing techniques that require a frequency comb that either spans a full octave or, in a more complicated scheme, ranges from a frequency $f$ to a frequency $3 / 2 f .7,8$ at the commonly used repetition rates near $1 \mathrm{GHz}$, femtosecond oscillators that cover such broad spectra have not been available. Microstructure fibers have been used for extracavity spectral broadening. ${ }^{9,10}$ Although these fibers have served well in the past, it has proved to be difficult to align the broad femtosecond laser spectrum into a sub-2- $\mu \mathrm{m}$ core. The input and output faces degrade after a certain time, and additional broadband amplitude noise can occur at higher pulse energies. ${ }^{3}$ More favorably, a directly octave spanning femtosecond laser has been achieved by Ell et al. ${ }^{11}$ Although here $f_{\text {CEO }}$ could be measured directly, with only $120 \mathrm{~mW}$ of output power and a $65-\mathrm{MHz}$ repetition rate, the rather complicated device does not yet appear optimal for frequency comb applications. In addition to their use in frequency metrology, femtosecond lasers that span an ultrabroad continuum to allow direct access to $f_{\text {CEO }}$ are of considerable interest for the synthesis of ultrashort pulses with a controlled carrier envelope phase evolution. Such devices are of particular importance for nonlinear experiments with carrier envelope phase sensitivity. This issue has been addressed by Krausz and co-workers, who used an external fiber for spectral broadening. ${ }^{12,13}$ In this Letter we report a new, extremely compact approach to achieving a broadband continuum from femtosecond oscillators at gigahertz repetition rates that permits direct access to $f_{\text {CEO }}$ without extracavity broadening. The concept is based on an increased self-amplitude modulation (SAM) of a pulse inside the cavity through the introduction of a slightly convex mirror into the collimated arm of our standard ring cavity. The requirements for accurate broadband intracavity GDD control are found to be greatly relaxed.

The standard bow-tie ring cavity used to achieve femtosecond pulses at repetition rates in the gigahertz range is sketched in Fig. 1 and has been described experimentally. ${ }^{1}$ It consists of a laser crystal located between two concave mirrors, M1 and M2, flat mirror M3, and flat output coupler OC. Following Ref. 14, we calculate the factor $\delta=\left(1 / w_{0}\right)\left(\partial w_{0} / \partial P\right)$, where $P$ is the intracavity peak power and $w_{0}$ is the beam radius at the waist inside the crystal. This factor reflects the power-induced modulation of the overlap between the intracavity beam and the pump volume mediated through the Kerr nonlinearity and thereby is a measure of the strength of the self-gain modulation of a circulating pulse. For stable mode locking, $\delta$ must be negative. This occurs at the inner edge of the stability map of a ring oscillator. In regions of the stability

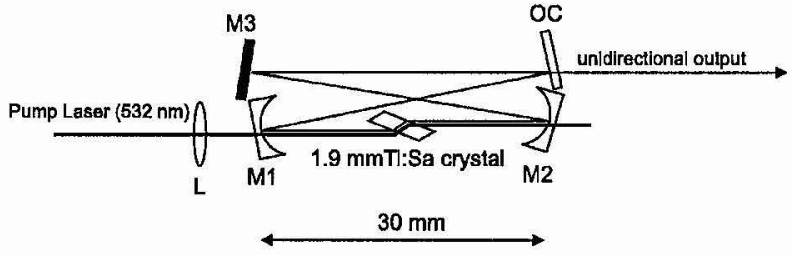

Fig. 1. The cavity consists of the two concave chirped mirrors, M1 and M2 (30-mm radius of curvature), mirror M3 (see text for details), and output coupler OC. The 1.9-mm-thick Ti:sapphire crystal is located at Brewster's angle between mirrors M1 and M2 and is pumped through lens $\mathrm{L}$ with a focal length of $30 \mathrm{~mm}$. 
map that permit stable mode locking, theory finds that replacing flat mirror M3 with a slightly convex mirror increases the absolute value of $\delta$. A radius of curvature of $1000 \mathrm{~mm}$, as was used in the experiment described below, results in an enhancement of $\delta$ by approximately a factor of 5. More illustratively, the gain increase induced by the Kerr lens can equivalently be considered an increased stationary gain plus a fast saturable absorber with a saturable absorption that amounts to the gain increase. Using a convex mirror instead of flat mirror M3 then leads to an increased modulation depth of the effective saturable absorber. Now, only a much shorter pulse can open a net gain window and maintain stable mode-locked operation. Stronger self-phase modulation and enhanced distribution of power to the spectral wings result. The model used to calculate $\delta$, however, is valid at intracavity peak powers below the critical power for self-focusing, $P_{C} \approx 960 \mathrm{~kW}$, only, whereas experimentally realized oscillators are usually above this threshold. Despite this, our proposal has been proved experimentally to be an intriguing way to enhance the SAM inside standard femtosecond laser cavities to facilitate broader spectra.

Except for mirror M3, the experimental setup is equivalent to the one described above (see Fig. 1). It contains a $1.9-\mathrm{mm}$ thick Brewster-angled Ti:sapphire crystal that is pumped through lens L (Fig. 1; focal length, $30 \mathrm{~mm}$ ) by $10.5 \mathrm{~W}$ of power from a Coherent Verdi laser. Mirror M3 has a convex surface with radius of curvature of $1000 \mathrm{~mm}$. Measured reflectivity data of chirped mirrors M1-M3 and of the output coupler are shown in Fig. 2, top. The highly reflecting band of the chirped mirrors ranges only from approximately 700 to $870 \mathrm{~nm}$. The losses out of this band amount to a few percent. The output coupler reflects $98 \%$ in the center of its band and rolls off to values much greater than $10 \%$ below approximately $680 \mathrm{~nm}$ and above $880 \mathrm{~nm}$. The bottom of Fig. 2 shows the net dispersion of the cavity described, taking into account the sapphire crystal, the chirped mirrors, and the output coupler. For the mirrors, only design data are available over the entire visible bandwidth and were considered. Below $730 \mathrm{~nm}$ the GDD is mostly positive, whereas above $850 \mathrm{~nm}$ strong oscillations with third-order dispersion of several thousands of $\mathrm{fs}^{3}$ are present. The oscillator is aligned to yield a repetition rate of $1 \mathrm{GHz}$. Output spectra were taken with a commercial spectrometer. Intensity autocorrelation traces of the pulses were measured with a second-harmonic autocorrelator after an extracavity pulse-compression stage that uses multiple reflections off of a pair of chirped mirrors.

Initially, the oscillator was aligned for maximum power in bidirectional continuous wave operation at the inner edge of the cavity stability range. To initiate mode locking we then translated M2 toward M1 until bidirectional pulsed operation occurred with two single counterpropagating pulses of 24 -fs duration (approximately $30-\mathrm{nm}$ bandwidth center at $800 \mathrm{~nm}$ ). Further translation of M2 toward M1 resulted in a strongly enhanced distribution of power to the spectral wings in discrete steps with intermediate modes of chaotic multipulse patterns and stable double pulses (spaced by approximately $100 \mathrm{fs}$ ). Finally, unidirectional mode locking with only one single circulating pulse occurred in a random direction, and a spectrum as shown in Fig. 3 was observed. Further translation of M2 stopped mode locking. At the final position of M2 the measured pulse duration was $12 \mathrm{fs}$ (assuming a $\operatorname{sech}^{2}$ envelope) optimized for the spectral part around $800 \mathrm{~nm}$. However, the reflectivities of the mirror and the output coupler greatly distorted the intracavity spectral shape; also, the positive GDD of the output coupler substrate could be compensated for only partially by the chirped mirrors. Therefore we believe that the intracavity pulse's duration is substantially shorter than the externally measurable value. The average power in the final position of M2 was $1.2 \mathrm{~W}$. Intersecting the pump or intracavity beam stopped mode locking, and the output power in both directions decreased by more than an order of magnitude to approximately $20-40 \mathrm{~mW}$.

The spectrum shown in Fig. 3 ranges from 560 to $1150 \mathrm{~nm}$ at $50 \mathrm{~dB}$ below its maximum at $664 \mathrm{~nm}$. Four distinct bands occur: a double-peaked structure

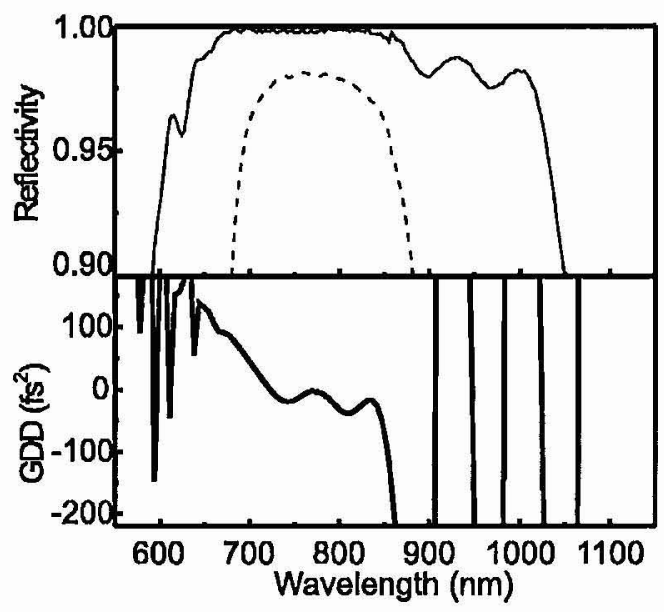

Fig. 2. Top, reflectivity of the chirped mirrors (solid curve) and of the output coupler (dashed curve). Bottom, net cavity GDD with the design values for the chirped mirrors and the output coupler as well as the dispersion of sapphire taken into account.

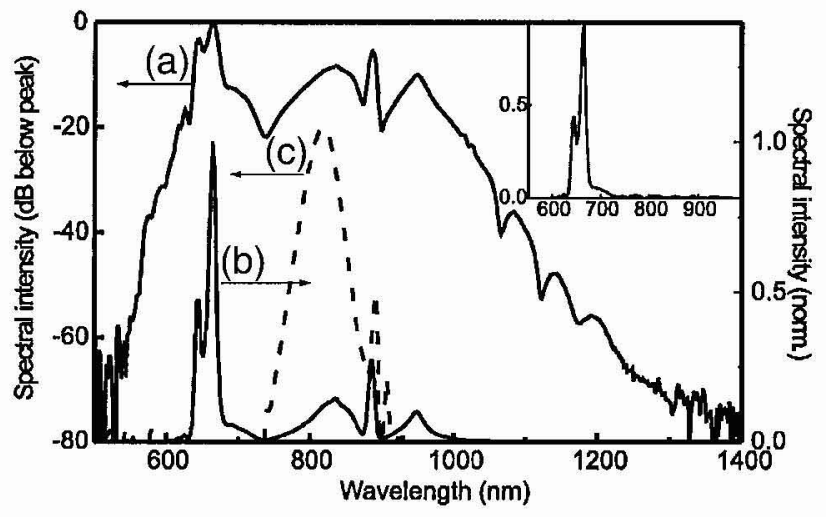

Fig. 3. Output spectrum in unidirectional operation on (a) a logarithmic and (b) a linear scale. The output spectrum of a laser with flat mirror M3 is shown offset by

$20 \mathrm{~dB}$ as curve (c). Inset, spectrum of the output after reflection off two high reflectors for $657 \mathrm{~nm}$. 
near $655 \mathrm{~nm}$, a broad feature with $62 \mathrm{~nm}$ FWHM at $835 \mathrm{~nm}$, and two peaks at $885 \mathrm{~nm}$ (FWHM, $10 \mathrm{~nm}$ ) and $950 \mathrm{~nm}$ (FWHM, $33 \mathrm{~nm}$ ). It should also be noted here that replacement of the convex mirror with a flat mirror resulted in a behavior that is equivalent to that of the laser described in Ref. 1; an output spectr um with that configuration is shown in Fig. 3.

Reflecting the output from a pair of high reflectors for $657-\mathrm{nm}$ wavelength with approximately $150-\mathrm{nm}$ bandwidth, a femtosecond pulse source near $655 \mathrm{~nm}$ with $\sim 450 \mathrm{~mW}$ of average power could be achieved. The spectrum behind the mirror pair is shown in the inset of Fig. 3. The 655-nm pulses were temporally characterized without external pulse compression and revealed a pulse length of $33 \mathrm{fs}$. To our knowledge, we have demonstrated the first femtosecond pulse source operating at this wavelength and far out of the amplification bandwidth of the gain material at a power level close to half of the spectrally integrated average power.

If pure solitonlike propagation were the dominant pulse-shaping mechanism, the net cavity dispersion should not support mode locked operation that exceeded an approximately $700-900-\mathrm{nm}$ band. In contrast, the only dispersion requirement for ultrabroadband operation has been found to be negative net GDD within the core region of the spectrum near $800 \mathrm{~nm}$. Adding positive GDD by using a longer crystal prevented pulsed operation, whereas an additional chirped mirror pair $\left(\approx 100 \mathrm{fs}^{2}\right.$ at $\left.800 \mathrm{~nm}\right)$ hardly changed the performance. This result indicates that only for a part of the spectrum do the GDD requirements for solitonlike pulse propagation need to be fulfilled to initiate mode locking. Considering the net cavity dispersion and the losses through mirrors at the spectral wings, further pulse shortening and subsequent spectral broadening by self-phase modulation can occur only during a few cavity round trips before the pulse exits the resonator. This is clearly related to the enhanced SAM induced by convex mirror M3. The theoretical prediction that increased saturable absorption will be responsible for the observed performance is also strongly supported by the drop in output power by more than an order of magnitude when mode locking is interrupted by intersection of the intracavity or pump beam.

In conclusion, we have demonstrated an extraordinarily compact five-element femtosecond Ti:sapphire oscillator emitting a broadband continuum with attractive prospects for application in future optical clockworks. At $50 \mathrm{~dB}$ a wavelength coverage from 560 to $1150 \mathrm{~nm}$ was achieved at a repetition rate of $1 \mathrm{GHz}$. In contrast to earlier approaches to ultrabroadband pulse generation, here a smooth negative intracavity dispersion is necessary only for a narrow core part of the spectrum. In collaborative experiments with the U.S. National Institute of Standards and Technology as reported elsewhere ${ }^{15}$ we have been able to demonstrate access to and control of $f_{\text {CEO }}$ without external spectral broadening. Therefore, we believe that, compared with state-of-the-art systems, the concept described here will permit more stable and compact clockworks for frequency metrology and future optical atomic clocks. The new technology can also be applied to gain precise control over the carrier envelope phase evolution of ultrashort pulses in a simpler and more stable setup. A strong distinct emission band at $655 \mathrm{~nm}$ occurs that can be extracted to become a powerful femtosecond pulse source at a wavelength that is beyond the gain spectrum of Ti:sapphire.

We thank P. Russbüldt (Institut für Lasertechnik, Aachen, Germany), S. A. Diddams and T. M. Ramond (National Institute of Standards and Technology, Boulder), and H. Giessen (Institut für Angewandte Physik, Bonn, Germany) for stimulating discussions and for their willingness to help us with essential equipment. This study has been funded by GigaOptics $\mathrm{GmbH}$, Germany. A. Bartels's e-mail address is bartels@iht-ii.rwth-aachen.de.

\section{References}

1. A. Bartels, T. Dekorsy, and H. Kurz, Opt. Lett. 24, 996 (1999).

2. Th. Udem, S. A. Diddams, K. R. Vogel, C. W. Oates, E. A. Curtis, W. D. Lee, W. M. Itano, R. E. Drullinger, J. C. Bergquist, and L. Hollberg, Phys. Rev. Lett. 86, 4996 (2001).

3. S. A. Diddams, Th. Udem, K. R. Vogel, C. W. Oates, E. A. Curtis, R. S. Windeler, A. Bartels, J. C. Bergquist, and L. Hollberg, in Laser Frequency Stabilization, Standards, Measurement, and Applications, J. L. Hall and J. Ye, eds., Proc. SPIE 4269, 77 (2001).

4. R. Holzwarth, Th. Udem, T. W. Hänsch, J. C. Knight, W. J. Wadsworth, and P. St. J. Russell, Phys. Rev. Lett. 85, 2264 (2000).

5. S. A. Diddams, Th. Udem, J. C. Bergquist, E. A. Curtis, R. E. Drullinger, L. Hollberg, W. M. Itano, W. D. Lee, C. W. Oates, K. R. Vogel, and D. J. Wineland, Science 293, 825 (2001).

6. J. Reichert, R. Holzwarth, Th. Udem, and T. W. Hänsch, Opt. Commun. 172, 59 (1999).

7. D. J. Jones, S. A. Diddams, J. K. Ranka, A. Stentz, R. S. Windeler, J. L. Hall, and S. T. Cundiff, Science 288, 635 (2000).

8. U. Morgner, R. Ell, G. Metzler, T. R. Schibli, F. X. Kärtner, J. G. Fujimoto, H. A. Haus, and E. P. Ippen, Phys. Rev. Lett. 86, 5462 (2001).

9. J. K. Ranka, R. S. Windeler, and A. J. Stenz, Opt. Lett. 25, 25 (2000).

10. J. C. Knight, T. A. Birks, P. St. J. Russell, and D. M. Atkin, Opt. Lett. 21, 1547 (1996).

11. R. Ell, U. Morgner, F. X. Kärtner, J. G. Fujimoto, E. P. Ippen, V. Scheuer, G. Angelow, T. Tschudi, M. J. Lederer, A. Boiko, and B. Luther-Davies, Opt. Lett. 26, 373 (2001).

12. L. Xu, Ch. Spielmann, A. Poppe, T. Brabec, F. Krausz, and T. W. Hänsch, Opt. Lett. 21, 2008 (1996).

13. A. Poppe, R. Holzwarth, A. Apolonski, G. Tempea, Ch. Spielmann, T. W. Hänsch, and F. Krausz, Appl. Phys. B 72, 373 (2001).

14. V. Magni, G. Cerullo, and S. de Silvestri, Opt. Commun. 96, 348 (1993).

15. T. M. Ramond, S. A. Diddams, L. Hollberg, and A. Bartels, Opt. Lett. 27, 1842 (2002). 